Classification

Physics Abstracts

$07.85-32.50-78.70$

\title{
A characteristic fluorescence correction factor for use in electron probe microanalysis. I. Theory
}

\author{
Christian Schiebl $\left({ }^{2}\right)$, Hans-Jürgen August $\left({ }^{1}\right)\left({ }^{\star}\right)$ and Johann Wernisch $\left({ }^{1}\right)$
}

(1) Institut für Angewandte und Technische Physik, Technische Universität Wien, Wiedner Hauptstr. 8-10, A-1040 Wien (Vienna), Austria

$\left({ }^{2}\right)$ Digital Equipment Corp., Campus-based Engineering Center, Favoritenstr. 7, A-1040 Wien (Vienna), Austria

(Received November 29, 1990; accepted March 21, 1991)

\begin{abstract}
A rigorous derivation of the characteristic fluorescence correction factor for use in electron probe microanalysis is presented. To achieve a high accuracy of the resulting expression Coster-Kronig transitions and effective fluorescence yields are taken into account and special attention is directed to the excitation conditions, e.g. the subshell structures. The integrations are carried out as accurately as possible, i.e. analytical solutions are employed if possible. Approximation and simplifications are avoided as far as possible.
\end{abstract}

\section{Introduction.}

Procedures for evaluating measurements in electron probe microanalysis often do without a correction of the fluorescence radiation excited by characteristic radiation or employ the "standard" correction developed by Reed [1]. Generally the contribution of secondary characteristic fluorescence is small compared to the total generated intensity, and therefore negligible. There are, however, cases where the characteristic fluorescence contribution is significant and requires consideration.

A number of investigators have proposed characteristic fluorescence corrections for thick specimens [see, e.g., 1-12]. However, some of these contributions to the problem discussed are quite old, so that partly very rough approximations were used, resulting in restrictions regarding the range of applicability.

More recent publications include more rigorous approaches, especially with respect to more realistic descriptions of the depth distribution function of primarily generated characteristic radiation. The accuracy of these models, though, is often spoiled due to the use of intensity ratios which generally are very rough and simplified representations of the real situation.

$\left.{ }^{\star}\right)$ Author to whom correspondance should be sent. 
Büchner and Stienen [10] have been the only investigators to take into account Coster-Kronig transitions, although - according to these authors - this type of transition may alter the intensity of the secondary fluorescence radiation by up to $30 \%$.

Moreover rough simplifications have been used concerning the conditions under which secondary fluorescence radiation is generated. Some older publications even neglect the subshell structure of the L-series.

The present paper deals with the rigorous derivation of a formula quantifying the secondary fluorescence correction in massive semi-infinite and homogeneous samples. The type of depth distribution of primarily generated characteristic X-radiation used is the one first proposed by Packwood and Brown [13], i.e. a modified Gaussian distribution. In order to obtain an expression as accurate as possible we considered Coster-Kronig transitions and relative fluorescence yields and directed special attention to the description of the excitation conditions. Simplifications and approximations are only employed if the resulting deviations are negligible with respect to the characteristic fluorescence correction factor.

\section{Derivation of the fluorescence correction factor.}

In the following the intensity of characteristic radiation of the detected line $i$ of element $j$ produced by ionizations caused by characteristic radiation $l$ of element $k$ shall be quantified. The sample under consideration is supposed to be semi-infinite. As basis of this derivation the quantification of generated primary radiation presented by Wernisch et al. [14] will be used. The generated intensity will generally be denoted by $n$, while the number of ionizations will be designated as $m$.

Figure 1 shows the geometry used for the derivation of the secondary fluorescence correction factor. The intensity $n_{p, k l}$ of primary radiation of element $k$ and line $l$ generated in the mass depth $\rho z$ is quantified by

$$
n_{p, k l}(\rho z) \mathrm{d} \rho z=m_{p, k}(\rho z) \omega_{k l} p_{k l} \mathrm{~d} \rho z
$$

with

$$
m_{p, k}(\rho z)=\frac{N_{0}}{A_{k}} c_{k} R_{k} / S_{k} \bar{\Phi}_{k}(\rho z)
$$

and

$$
\bar{\Phi}_{k}(\rho z)=\frac{\Phi_{k}(\rho z)}{\int_{0}^{\infty} \Phi_{k}(\rho z) \mathrm{d} \rho z}
$$

$m$ stands for the amount of ionizations, $\omega$ is the fluorescence yield, $p$ the transition probability, and $N_{0}$ is Avogradro's constant. $A$ stands for the atomic number of the element in question and $c$ for its weight fraction, while $R$ is the backscattering factor and $1 / S$ the stopping power factor. $\Phi$ is the symbol for the depth distribution function, i.e. the function quantifying the dependence of radiation generation on mass depth.

Since the primarily generated photons are partly absorbed on their way from the point of generation to the point where they cause secondary fluorescence, only a part of this primary radiation arrives at the surface of a sphere having a radius $\rho r$ :

$$
n_{p, k l}(\rho z, \rho r) \mathrm{d} \rho z=n_{p, k l}(\rho z) \exp \left(-\chi_{k l} \rho r\right) \mathrm{d} \rho z
$$

with

$$
\chi_{k l}=\sum_{n} c_{n}\left(\mu_{k l, n} / \rho_{n}\right)
$$




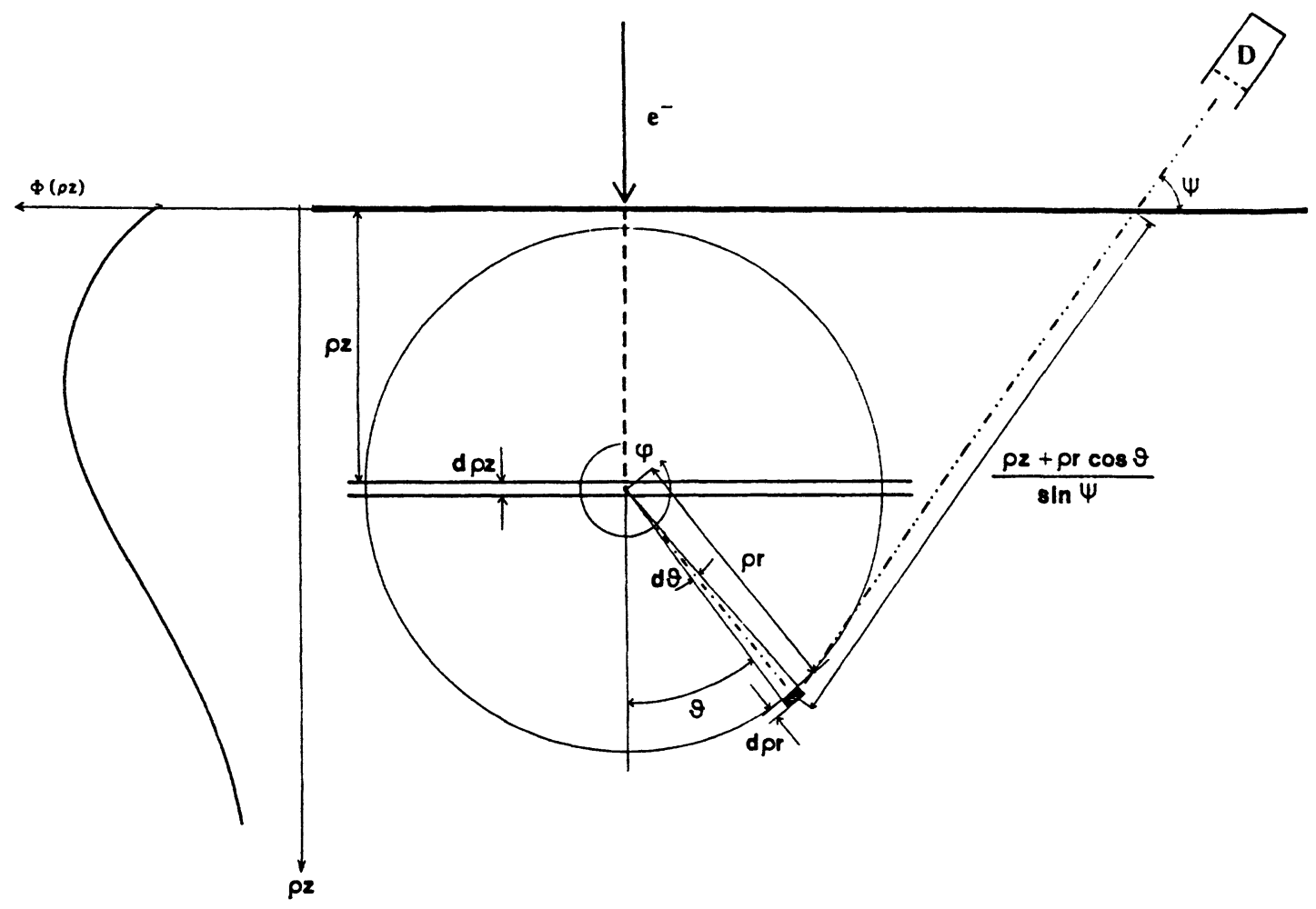

Fig. 1. - Scheme of the geometry used for the derivation of the secondary fluorescence factor.

where $\mu_{k l, n} / \rho_{n}$ is the mass absorption coefficient for radiation of type $l$ of the element $k$ absorbed in element $n$.

To calculate the secondary fluorescence correction factor it must be known how many ionizations are caused at the distance $\rho z$ from the point from which the primary radiation originates. For this the amount of primary radiation absorbed due to photoionization processes between $\rho r$ and $\rho r+\Delta \rho r$ must be quantified.

$$
n_{p, k l}(\rho z, \rho r) \mathrm{d} \rho z-n_{p, k l}(\rho z, \rho r) \exp \left[-c_{j}\left(\tau_{k l, j} / \rho_{j}\right) \Delta \rho r\right] \mathrm{d} \rho z
$$

In the case $\Delta \rho r \rightarrow 0$ we obtain

$$
n_{p, k l}(\rho z, \rho r) c_{j}\left(\tau_{k l, j} / \rho_{j}\right) \mathrm{d} \rho z \mathrm{~d} \rho r
$$

where $\tau_{k l, j} / \rho_{j}$ is the photoabsorption coefficient for the radiation of type $l$ of element $k$ in element $j$.

Until now the angle dependence of the secondary ionizations has not been taken into account. Allowing for this, the amount of secondary radiation $n_{s, j i}$ generated in the volume $\sin (\vartheta) \mathrm{d} \vartheta \mathrm{d} \varphi \mathrm{d} \rho r$ can be quantified by

$$
n_{s, j i}(\rho z, \rho r, \vartheta, \varphi) \mathrm{d} \vartheta \mathrm{d} \varphi \mathrm{d} \rho r \mathrm{~d} \rho z=n_{p, k l}(\rho z, \rho r) c_{j}\left(\tau_{k l, j} / \rho_{j}\right) \frac{r_{j i}-1}{r_{j i}^{\prime}} \omega_{j i} p_{j i} \frac{\sin (\vartheta)}{4 \pi} \mathrm{d} \varphi \mathrm{d} \vartheta \mathrm{d} \rho z \mathrm{~d} \rho r
$$


where $\frac{r_{j i}-1}{r_{j i}^{\prime}}$ is the jump ratio of the absorption edge.

Finally the absorption of a part of the secondary radiation on its way from the point of generation to the surface of the sample must be taken into account. Of course the detector registers only a small part of the radiation leaving the sample, depending on the solid angle $\Omega$ covered by the detector.

Henceforward the lateral distribution of the secondary radiation generation shall be neglected. In this case the absorption does not depend on the angle $\varphi$, and the integration over $\varphi$ simply yields $2 \pi$.

The generation mass depth of secondary radiation is quantified by

$$
\rho z+\rho r \cos (\vartheta)
$$

and thus the distance travelled by the quanta in the sample towards the detector is

$$
\frac{\rho z+\rho r \cos (\vartheta)}{\sin (\psi)}
$$

where $\psi$ is the take-off angle of the detected radiation.

Thus the corresponding absorption is quantified by the term

$$
\exp \left[-\chi_{j i}(\rho z+\rho r \cos \vartheta)\right]
$$

with

$$
\chi_{j i}=\sum_{n} c_{n}\left(\mu_{j i, n} / \rho_{n}\right) \frac{1}{\sin (\psi)}
$$

Summarizing the results obtained so far and taking into account the whole intensity of secondary radiation of line $i$ of element $j$ caused by ionizations due to radiation of line $l$ of element $k$ we obtain an expression for the secondary fluorescence intensity $n_{s, j i}^{\#}$ arriving at the detector:

$$
\begin{aligned}
n_{s, j i}^{\#} & =\iiint n_{s, j i}^{\#}(\rho z, \rho r, \vartheta) \mathrm{d} \vartheta \mathrm{d} \rho r \mathrm{~d} \rho z \\
& =\frac{1}{2} \frac{N_{0}}{A_{k}} R_{k l} / S_{k l} c_{k} c_{j} \omega_{k l} p_{k l} \omega_{j i} p_{j i}\left(\tau_{k l, j} / \rho_{j}\right) \frac{r_{j i}-1}{r_{j i}^{\prime}} \frac{\Delta \Omega}{4 \pi} f_{k l, j}
\end{aligned}
$$

with

$$
\left.f_{k l, j i}=\iiint \bar{\Phi}_{k l} \rho z\right) \exp \left(-\chi_{k l} \rho r\right) \exp \left[-\chi_{j i}(\rho z+\rho r \cos \vartheta)\right] \sin (\vartheta) \mathrm{d} \vartheta \mathrm{d} \rho r \mathrm{~d} \rho z
$$

To obtain the fluorescence correction factor for the radiation of line $i$ of element $j$ the ratio of secondary radiation to the primary radiation must be calculated. The amount of primary radiation, i.e. radiation generated by electron impact, of the detected line $i$ of element $j$ is obtained by

$$
n_{p, j i}^{\#}=\frac{N_{0}}{A_{j}} R_{j i} / S_{j i} c_{j} \omega_{j i} p_{j i} \frac{\Delta \Omega}{4 \pi} f_{j i}\left(\chi_{j i}\right)
$$

Assuming the most general case, in which several lines of several elements in the sample can cause ionizations resulting in radiation of line $i$ of element $j$, a summation of all lines $l$ of all elements $k$ capable of causing ionizations must be carried out. Thus the fluorescence correction factor $F_{j i}$ results in:

$$
\begin{aligned}
F_{j i} & =1+\frac{\sum \sum n_{s, j i}^{\#}}{n_{p, j i}} \\
& =1+\frac{1}{2} \frac{A_{j}}{R_{j i}} \frac{1}{1 / S_{j i}} \frac{r_{j i}-1}{r_{j i}^{\prime}} \omega_{j i} p_{j i} \frac{1}{f_{j i}\left(\chi_{j i}\right)} \sum_{k} \frac{c_{k}}{A_{k}} \sum_{l} \frac{R_{k l}}{S_{k l}} \frac{\omega_{k l}}{\omega_{j i}} \frac{p_{k l}}{p_{j i}}\left(\frac{\tau_{k j}}{\rho_{j}}\right) f_{k l, j i}
\end{aligned}
$$


2.1 INTEGRATION. - Since the geometry applied for the derivation of the secondary fluorescence correction factor is independent of the angle $\varphi$ the evaluation of the integrals of equation $(9)$ is reduced to a two-dimension problem.

First the factor $f_{k i, j i}$ shall be represented as

$$
f_{k l, j i}=\int \bar{\Phi}_{k l}(\rho z) f(\rho z) \mathrm{d} \rho z
$$

and the quantity

$$
f(\rho z)=\exp \left(-\chi_{j i} \rho z\right) \iint \exp \left(-\chi_{k l} \rho r\right) \exp \left(-\chi_{j i} \rho r \cos \vartheta\right) \sin (\vartheta) \mathrm{d} \vartheta \mathrm{d} \rho r
$$

shall be calculated.

For the integration the two regions

region A: $\rho r \leq \rho z$ and

region B: $\rho r>\rho z$

must be distinguished. Region $\mathrm{B}$ must be further divided into

region $\mathrm{B} 1: \rho z_{1} \geq \rho z$ and

region $\mathrm{B} 2: \rho z_{1}<\rho z$,

where $\rho z_{1}$ is the absolute mass depth of secondary radiation generation. The principe of this region distinction is shown in figure 2 .

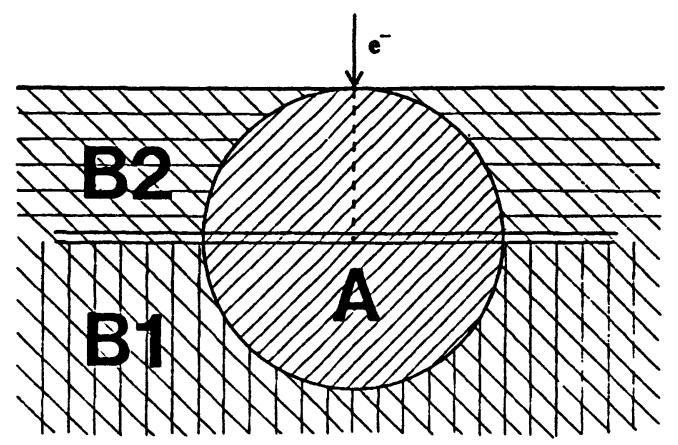

Fig. 2. - Integration regions used in the present model.

In case of regions $\mathrm{A}$ and $\mathrm{B} 1$ the integration over $\vartheta$ can be carried out analytically. In a generalized form this integration can be represented by

$$
\int_{0}^{a} \exp [-\lambda \cos (\vartheta)] \sin (\vartheta) \mathrm{d} \vartheta=\left.\frac{1}{\lambda} \exp [-\lambda \cos (\vartheta)]\right|_{0} ^{a}
$$

where $a$ equals $\pi$ in the case of region A and $\pi / 2$ in the case of region B1.

The integration over the mass thickness $\rho r$ leads to the first exponential integral:

$$
\int_{a}^{b} \frac{\exp (\lambda x)}{x} \mathrm{~d} x
$$


In the case of region A $a$ equals $0, b$ equals $\rho z$ and $\lambda$ can be either smaller or larger than 0 . For region B1 $a$ equals $\rho z, b$ equals $\infty$ and $\lambda$ is always smaller than 0 .

The exponential integral cannot be solved analytically, unless it is transformed into a series expansion. In region $\mathrm{A}$, moreover two different cases must be distinguished, namely $\lambda<0$ and $\lambda>0, \lambda$ being $\chi_{j i}-\chi_{k l}$. Thus we arrive at

$$
f^{(\mathrm{A} \text { and } \mathrm{B} 1)}(\rho z)=\frac{\exp \left(-\chi_{j i} \rho z\right)}{\chi_{j i}}\left[\ln \left(\frac{\chi_{k l}+\chi_{j i}}{\chi_{k l}}\right)+\sum_{n=1}^{\infty} \frac{\delta}{n n !} \rho z^{n}\right]
$$

with

$$
\begin{array}{ll}
\delta=(-1)^{n+1} \chi_{k l}^{n}+\left(\chi_{j i}-\chi_{k l}\right)^{n} & \text { for } \chi_{j i}>\chi_{k l} \\
\delta=(-1)^{n+1}\left[\chi_{k l}^{n}-\left(\chi_{k l}-\chi_{j i}\right)^{n}\right] & \text { for } \chi_{i j}<\chi_{k l}
\end{array}
$$

In region B2 the upper limit of the integral over $\rho r$ is a function depending on the angle $\vartheta$.

The sequence of the integrations therefore has to be changed.

The first integral to be evaluated has the general form

$$
\int \exp (-\lambda x) \mathrm{d} x=\left.\frac{1}{\lambda} \exp (-\lambda x)\right|_{\rho z} ^{\rho z / \cos (\pi-\vartheta)}
$$

Since the integral over $\rho r$ cannot be evaluated analytically it shall be left uncalculated for the present. In region $\mathrm{B} 2$ we thus obtain:

$$
\begin{gathered}
f^{(\mathrm{B} 2)}(\rho z)=\exp \left(-\chi_{j i} \rho z\right) \int_{\pi / 2}^{\pi} \frac{\sin (\vartheta)}{\chi_{k l}+\chi_{j i} \cos (\vartheta)}\left\{\exp \left[-\left(\chi_{k l}+\chi_{j i} \cos \vartheta\right) \rho z\right]\right. \\
\left.-\exp \left[-\left(\chi_{k l}+\chi_{j i} \cos \vartheta\right) \frac{\rho z}{\cos (\pi-\vartheta)}\right]\right\} \mathrm{d} \vartheta
\end{gathered}
$$

For the depth distribution function of primary radiation the model first presented by Packwood and Brown [13] shall be employed. This model reproduces the distribution of primarily generated $\mathrm{X}$-rays in depth following a modified Gaussian distribution:

$$
\Phi(\rho z)=[\gamma-[\gamma-\Phi(0)] \exp (-\beta \rho z)] \exp \left[-(\alpha \rho z)^{2}\right]
$$

Using this very often used [15-24] general description of the depth distribution function the remaining integrals over $\rho z$ (see Eq. (13)) in region A and B1 can only be carried out numerically.

In region $B 2$ the sequence of integration can be changed in order to calculate the integral over $\rho z$ before that over $\vartheta$. This leads to integrals of the following general form:

$$
\int_{0}^{\infty} \exp (-s x) \exp \left(-d x^{2}\right) \mathrm{d} x=\frac{1}{2} \sqrt{\pi / d} \exp \left[\frac{s^{2}}{4 d}\right] \operatorname{erfc}\left[\frac{s}{2 \sqrt{d}}\right]
$$

with

$$
\operatorname{erfc}(x)=1-\operatorname{erf}(x)
$$

$\operatorname{erf}(x)$ being the error function. Several approximations for this type of function are known in literature [25].

Summarizing it can be stated that the factor $f_{k l, j i}$ can be calculated mainly analytically, although the remaining integrations over $\rho z$ (in regions A and B1) and $\vartheta$ (in region B2), respectively, 
have to be evaluated numerically. If, according to several approximations, the error function is represented as

$$
\operatorname{erf}(x)=1-R(x) \exp \left(-x^{2}\right)
$$

then the results of the calculations discussed above can be summarized as follows:

$$
\begin{gathered}
f_{k l, j i}=f_{k j, j i}^{(\mathrm{A}+\mathrm{B} 1)}+f_{k l, j i}^{(\mathrm{B} 2)} \\
f_{k l, j i}^{(\mathrm{A}+\mathrm{B} 1)}=\frac{1}{\chi_{j i}}\left[\ln \left(\frac{\chi_{k l}+\chi_{j i}}{\chi_{k l}}\right)\left(\frac{\gamma \sqrt{\pi}}{2 \alpha} R\left[\frac{\chi_{j i}}{2 \alpha}\right]-[\gamma-\Phi(0)] \frac{\sqrt{2}}{\alpha} R\left[\frac{\chi_{j i}+\beta}{2 \alpha}\right]\right)+\right. \\
+\int_{0}^{\infty} \sum_{n=1}^{\infty} \frac{\delta}{n n !} \rho z^{n}\{\gamma-[\gamma-\Phi(0)] \exp (-\beta \rho z)\} \exp \left(-\chi_{j i} \rho z\right) \exp \left[-(\alpha \rho z)^{2}\right] \mathrm{d} \rho z
\end{gathered}
$$

with

$$
\begin{array}{rll}
\delta & =(-1)^{n+1} \chi_{k l}^{n}+\left(\chi_{j i}-\chi_{k l}\right)^{n} & \text { for } \chi_{j i}>\chi_{k l} \\
\delta & =(-1)^{n+1}\left[\chi_{k l}^{n}-\left(\chi_{k l}-\chi_{j i}\right)^{n}\right] & \text { for } \chi_{j i}<\chi_{k l}
\end{array}
$$

with

$$
\begin{aligned}
& b_{1}=\chi_{k l}+\chi_{j i}[1+\cos (\vartheta)] \\
& b_{2}=\frac{\chi_{k l}+\chi_{j i} \cos (\vartheta)}{\cos (\pi-\vartheta)}+\chi_{j i} \\
& b_{3}=\chi_{k l}+\chi_{j i}[1+\cos (\vartheta)]+\beta \\
& b_{4}=\frac{\chi_{k l}+\chi_{j i} \cos (\vartheta)}{\cos (\pi-\vartheta)}+\chi_{j i}+\beta
\end{aligned}
$$

2.2 CONDITIONS FOR EXCITATIONS OF SECONDARY FLUORESCENCE. - The radiation of all lines whose radiation energy is larger than the edge energy of a given shell is in principle capable of ionizing this shell. Since, on the other hand, the consideration of all possible lines would lead to time-consuming and complex calculations, some simplifications shall be introduced, which will be discussed hereafter.

If the ionizing radiation originates from the K-shell, then the excitation of the $\mathrm{K}_{\alpha 1}$ and of the $\mathrm{K}_{\alpha 2}$-lines are calculated using the energy of the former line. The excitation due to $\mathrm{K}_{\beta 1}$-radiation is also taken into account, and the sum of these intensities is normalized to unity. The excitation caused by weaker lines, e.g. $\mathrm{K}_{\beta 2}, \mathrm{~K}_{\beta 3}$ etc. is not calculated explicitely, but through the normalization mentioned above also these contributions are taken into account, though assuming that all radiation energies of the $\beta$-series are equal to that of the $\mathrm{K}_{\beta 1}$-line.

In case of excitation through $\mathrm{L}$-lines the structure of the L-subshells $\left(\mathrm{L}_{1}, \mathrm{~L}_{2}\right.$ and $\mathrm{L}_{3}$, the latter showing the highest edge energy) has to be allowed for. The transitions of electrons ending on a L-subshell have different probabilities. In case of transitions ending on the $\mathrm{L}_{3}$-subshell those starting from the $\mathrm{M}_{4}$ and $\mathrm{M}_{5}$-subshells, designated as $\mathrm{L}_{\alpha 1}$ - and $\mathrm{L}_{\alpha 2}$-lines, respectively, are the most probable ones, the intensity of the $\mathrm{L}_{\alpha 1}$-line being about ten times larger than that of the $\mathrm{L}_{\alpha 2}$-line. 
That is why all lines resulting from transitions ending on the $\mathrm{L}_{3}$-subshell shall be regarded as having the energy of the $\mathrm{L}_{\alpha 1}$-line.

The strongest line resulting from transitions ending on the $\mathrm{L}_{2}$-subshell is the one designated as $\mathrm{L}_{\beta 1}$. All lines produced by transitions on the $\mathrm{L}_{2}$-subshell shall therefore be regarded as having the same energy as this line. Analogously transitions ending on the $\mathrm{L}_{1}$-subshell shall be considered as producing lines having the $\mathrm{L}_{\beta 3}$-line energy.

It should be mentioned that the transitions ending on the $\mathrm{L}_{3}$-subshell lead to the strongest lines, followed by those ending on the $\mathrm{L}_{2}$-subshell, while the transitions to the $\mathrm{L}_{1}$-subshell only cause very weak lines. The errors introduced by neglecting the latter lines is, therefore, very small.

The situation concerning the M-lines is, due to a more complex subshells' structure, more complicated. Since the secondary fluorescence caused by these lines is generally quite small, and since moreover many important features of these lines are not well known, we decided to assume a monochromatic line having the energy of the $\mathrm{M}_{\alpha 1,2}$-line.

To calculate the fluorescence correction factor according to the model presented above it is also necessary to take into account intershell-transitions, i.e. radiationless transitions between two subshells. These transitions, usually called Coster-Kronig-transitions, shall be discussed in detail in the next section.

2.3 COSTER-KRONIG-TRANSITIONS AND EFFECTIVE FLUORESCENCE YIELDS. - To describe the physical situation leading to secondary fluorescence effects as realistically as possible it is necessary to take into account Coster-Kronig-transitions. Büchner and Stienen [10] reported that the effect of Coster-Kronig-transitions can change the secondary fluorescence intensity of a given line by up to $30 \%$, thus justifying a more thorough investigation of these phenomena.

The effect of radiationless transitions shall be taken into account by means of effective fluorescence yields as known from literature [26]. We will, therefore, further discuss the term

$$
\left(\frac{r_{j i}-1}{r_{j i}^{\prime}} \omega_{j i} \mathrm{p}_{j i}\right) \frac{\omega_{k l} p_{k l}}{\omega_{j i} p_{j i}}
$$

of the fluorescence correction factor (see Eq. (12)). The factor $\omega_{j i} p_{j i}$ in the denominator results from the normalization of the primary generated radiation intensity and corresponds, therefore, to the line used for the evaluation of the measurements, i.e. in general to an $\alpha$-line.

In case of the $\mathrm{K}_{\alpha}$-line we obtain:

$$
\omega_{j i}=\omega_{\mathrm{K}}^{j} \quad \text { and } \quad p_{j i}=\frac{I\left(\mathrm{~K}_{\alpha}\right)}{I\left(\mathrm{~K}_{\alpha}+\mathrm{K}_{\beta}\right)}
$$

where $I$ of course stands for the respective intensities.

In case of an $\mathrm{L}_{\alpha}$-line being measured, only the ionizations of the $\mathrm{L}_{3}$-subshell are relevant, although the structure of the subshells has to allowed for. We assume, furthermore, that the energy of the electrons is higher than the edge energy of the $\mathrm{L}_{1}$-subshell, which means that all three subshells are supposed to be ionized. This assumption is surely valid if the $\mathrm{L}_{\alpha}$-line is used for the evaluation of the measurement data, and only in this case a fluorescence correction will be needed. For the effective fluorescence yield and the relative line intensity we thus obtain:

$$
\begin{aligned}
\omega_{j i}^{\text {eff }} & =\left[1+\frac{N_{2}}{N_{3}} f_{23}+\frac{N_{1}}{N_{3}}\left(f_{13}+f_{12} f_{23}\right)\right] \omega_{\mathrm{L}_{3}}^{j} \\
p_{j i} & =\frac{I\left(\mathrm{~L}_{\alpha}\right)}{I\left(\text { all lines resulting from transitions ending on } \mathrm{L}_{3}\right)}
\end{aligned}
$$


The quantity $f_{x y}$ is the Coster-Kronig transition probability, i.e. the probability of an innershell transition from subshell $\mathrm{L}_{y}$ to subshell $\mathrm{L}_{x}$.

$N_{i}$ stands for the relative frequency of primarily produced vacancies in the subshell $\mathrm{L}_{i}$. Heinrich [26] proposes to calculate in first order these vacancies according to the number of electrons in the respective subshells, i.e.

$$
N_{1}: N_{2}: N_{3}=1: 1: 2
$$

Fink et al. [27] quantify this relation in a slightly different way. Based on the ionization cross section of Bethe [28] they established the relation

$$
N_{1}: N_{2}: N_{3}=\frac{1}{E_{1}}: \frac{1}{E_{2}}: \frac{2}{E_{3}}
$$

where $E_{1,2,3}$ are the edge energies of the respective subshells.

The factor $\omega_{k l} p_{k l}$ is related to the exciting line, i.e. the primary generated radiation. If this is a $\mathrm{K}$-line, the $\mathrm{K}_{\alpha}-$ and $\mathrm{K}_{\beta}$-contribution are taken into account separately. This results in:

$$
\begin{array}{ll}
\omega_{k l}=\omega_{\mathrm{K}}^{k} \quad \text { and } \quad p_{k l}=\frac{I\left(\mathrm{~K}_{\alpha}\right)}{I\left(\mathrm{~K}_{\alpha}+\mathrm{K}_{\beta}\right)} \text { for } \mathrm{K}_{\alpha} \text {-lines } \\
\omega_{k l}=\omega_{\mathrm{K}}^{k} \quad \text { and } \quad p_{k l}=\frac{I\left(\mathrm{~K}_{\beta}\right)}{I\left(\mathrm{~K}_{\alpha}+\mathrm{K}_{\beta}\right)} \text { for } \mathrm{K}_{\beta} \text {-lines }
\end{array}
$$

For the description of L-lines as exciting radiations the following assumptions are made: First, the electron acceleration energy is large enough to make possible an ionization of all L-subshells, and, second, all lines resulting from transitions ending on an L-subshell are treated as being monochromatic, having the energy of the strongest line. The second assumption leads to the value 1 for the relative intensities. The fluorescence yields are calculated as follows:

$$
\begin{array}{ll}
\mathrm{L}_{1} \text {-subshell : } & \omega_{k l}=\omega_{\mathrm{L}_{1}}^{k} \\
\mathrm{~L}_{2} \text {-subshell : } & \omega_{k l}=\left(1+N_{1} / N_{2} f_{12}\right) \omega_{\mathrm{L}_{2}}^{k} \\
\mathrm{~L}_{3} \text {-subshell : } & \omega_{k l}=\left[1+N_{2} / N_{3} f_{23}+N_{1} / N_{3}\left(f_{13}+f_{12} f_{23}\right)\right] \omega_{\mathrm{L}_{3}}^{k}
\end{array}
$$

Finally the factor $\frac{r_{j i}-1}{r_{j i}^{\prime}} \omega_{j i} p_{j i}$ shall be discussed in detail. This quantity is related to the secondary radiation. First the ionization probability with respect to secondary fluorescence shall be quantified. In case of K-shells this result in:

$$
Q_{\mathrm{K}} \propto(\mu / \rho)_{\mathrm{K}}=\left[\frac{r_{\mathrm{K}}-1}{r_{\mathrm{K}}}\right](\mu / \rho)_{\text {total }}
$$

In case of L-subshells we obtain for $E_{\text {char }}>E_{\mathrm{L}_{1}}$ :

$$
\begin{array}{ll}
\mathrm{L}_{1} \text {-subshell : } & Q_{\mathrm{L}_{1}} \propto(\mu / \rho)_{\mathrm{L}_{1}}=\left[\frac{r_{\mathrm{L}_{1}}-1}{r_{\mathrm{L}_{1}}}\right](\mu / \rho)_{\text {total }} \\
\mathrm{L}_{2} \text {-subshell : } & Q_{\mathrm{L}_{2}} \propto(\mu / \rho)_{\mathrm{L}_{2}}=\left[\frac{r_{\mathrm{L}_{2}}-1}{r_{\mathrm{L}_{2}}}\right] \frac{1}{r_{\mathrm{L}_{1}}}(\mu / \rho)_{\text {total }} \\
\mathrm{L}_{3} \text {-subshell : } & Q_{\mathrm{L}_{3}} \propto(\mu / \rho)_{\mathrm{L}_{3}}=\left[\frac{r_{\mathrm{L}_{3}}-1}{r_{\mathrm{L}_{3}}}\right] \frac{1}{r_{\mathrm{L}_{1}} r_{\mathrm{L}_{2}}}(\mu / \rho)_{\text {total }}
\end{array}
$$


The respective total intensities caused by transitions ending at the three subshells thus can be represented as follows:

$$
\begin{aligned}
& I_{\mathrm{L}_{1}} \propto Q_{\mathrm{L}_{1}} \omega_{\mathrm{L}_{1}} \\
& l_{\mathrm{L}_{2}} \propto\left[Q_{\mathrm{L}_{2}}+f_{12} Q_{\mathrm{L}_{1}}\right] \omega_{\mathrm{L}_{2}} \\
& l_{\mathrm{L}_{3}} \propto\left[Q_{\mathrm{L}_{3}}+f_{23} Q_{\mathrm{L}_{2}}+\left(f_{13}+f_{12} f_{23}\right) Q_{\mathrm{L}_{1}}\right] \omega_{\mathrm{L}_{3}}
\end{aligned}
$$

These relations can easily be evaluated using the formulae derived in equation (33).

For $\mathrm{K}_{\alpha}$-radiation this result in:

$$
\frac{r_{j i}-1}{r_{j i}^{\prime}} \omega_{j i}=\left(\frac{r_{\mathrm{K}}^{j}-1}{r_{\mathrm{K}}^{j}}\right) \omega_{\mathrm{K}}^{j} \quad p_{j i}=\frac{l\left(\mathrm{~K}_{\alpha}\right)}{l\left(\mathrm{~K}_{\alpha}+\mathrm{K}_{\beta}\right)}
$$

and in the case of $\mathrm{L}_{\alpha}$-radiation (only $\alpha$-radiation shall be considered for the analysis) in:

$$
\begin{aligned}
& E_{\mathrm{L}_{2}, j}>E_{\text {char }, k}>E_{\mathrm{L}_{3}, j}: \\
& \frac{r_{j i}-1}{r_{j i}^{\prime}} \omega_{j i}=\left(\frac{r_{3}^{j}-1}{r_{3}^{j}}\right) \omega_{\mathrm{L}_{3}}^{j} \\
& E_{\mathrm{L}_{1, j}}>E_{\text {char }, k}>E_{\mathrm{L}_{2}, j:} \\
& \frac{r_{j i}-1}{r_{j i}^{\prime}} \omega_{j i}=\left[\left(\frac{r_{3}^{j}-1}{r_{3}^{j}}\right) \frac{1}{r_{2}^{j}}+f_{23}\left(\frac{r_{2}^{j}-1}{r_{2}^{j}}\right)\right] \omega_{\mathrm{L}_{3}}^{j} \\
& E_{\text {char }, k}>E_{\mathrm{L}_{1}, j}: \\
& \frac{r_{j i}-1}{r_{j i}^{\prime}} \omega_{j i}=\left[\left(\frac{r_{3}^{j}-1}{r_{3}^{j}}\right) \frac{1}{r_{2}^{j}} \frac{1}{r_{1}^{j}}+f_{23}\left(\frac{r_{2}^{j}-1}{r_{2}^{j}}\right) \frac{1}{r_{1}^{j}}+\left(f_{13}+f_{12} f_{23}\right)\left(\frac{r_{1}^{j}-1}{r_{1}^{j}}\right)\right] \omega_{\mathrm{L}_{3}}^{j}
\end{aligned}
$$

From equation (36) it can be easily seen that the absorption edge jump ratio depends on the energy of the exciting radiation $l$ of element $k$.

\section{Conclusions.}

The calculation of the secondary fluorescence correction factor presented in this paper directs special attention to an accurate consideration of the excitation conditions, Coster-Kronig transitions and the effective fluorescence yields. In order not to spoil the accuracy of the resulting correction factor rough approximations, such as in the older approaches, are completely avoided. Integrals are solved either analytically or using highly accurate approximations. The rigorous mathematical treatment of the problem ensures a high accuracy of the resulting fluorescence correction factor which is a considerable improvement over the theories quantifying secondary fluorescence presented so far.

Considerable further improvements are expected not to result from a further development of the basic theory of secondary radiation generation, but rather from improvements of basic quantities necessary for calculating the secondary fluorescence factor, e.g. more accurate quantifications of fluorescence yields and Coster-Kronig transition probabilities, more realistic representations of the depth distribution of primarily generated characteristic radiation, etc. Such aspects of fluorescence correction are of course independent of the basic theory presented in this paper, but rather depend on further experimental investigations of the respective basic quantities. The extension of the theory with respect to M-lines has not been presented here because of the lack 
of really reliable basic data required for the evaluation of the fluorescence correction factor, e.g. with reference to fluorescence yields, Coster-Kronig transition probabilities or even ionization cross sections. Nevertheless the principles of the theory presented hold of course also in the case of M-lines, and suitable modifications can be easily obtained.

A comprehensive survey of application examples and results of our calculations will be presented in a forthcoming paper along with comparisons with other correction procedures known so far.

\section{Acknowledgements.}

The authors acknowledge the support of the Austrian Fonds zur Förderung der wissenschaftlichen Forschung (Projekt P 7336 Phy).

\section{References}

[1] ReEd S. J. B., Brit. J. Appl. Phys. 16 (1965) 913-26.

[2] CASTAING R. Ph. D Thesis, University of Paris (ONERA Publ. Nr.) (1951) 55.

[3] WrTtry D. B., Ph. D. Thesis, California Institute of Technology, Pasadena, California (1957).

[4] BIRKS L. S., J. Appl. Phys. 32 (1961) 387.

[5] BIRKS L. S. Spectrochim Acta 17 (1961) 148.

[6] WITTRY D. B. University of Southern California Engeneering Center, Report 84-204 (1962).

[7] COLBY J. W. USAEC Report NLCO-917 (1964).

[8] CRISS J. and BIRKS L. S., in: The Electron Microprobe, McKinley T. D. Heinrich K. F. J. and WITTRY T. D. Eds. (John Wiley \& Sons, New York, 1966) 217.

[9] CRISS J. in: Quantitative Electron Microanalysis, Heirich K. F. J. Eds., NBS Special Publ. 298, National Bureau of Standards, U. S. Dept. of Commerce, Washington, D. C. (1968) 53.

[10] BüCHNER A. R., and STIENEN J. P. M., Mikrochim. Acta, Suppl. 6 (1975) 227-50.

[11] ARMSTRONG J. T. and BUSECK P. R. X-ray Spectrom. 14 (1985) 172-82.

[12] Ugarte D., Castellano G., Trincavelli J. Del Giorgio M. Riveros J. A. X-ray Spectrom. 16 (1987) 249-54.

[13] PACKWOOD R. H. and BROWN J. D., X-ray Spectrom. 10 (1981) 138-46.

[14] WeRnisCH J., PÖHN Ch., HANKe W. and EBEL H. X-ray Spectrom. 13 (1984) 180-1.

[15] BROWN J. D. and PACKWOOD R. H., X-ray Spectrom. 11 (1982) 187-93.

[16] BASTIN G. F., VAN LOO F. J. J. and HEIJLIGERS H. J. M. X-ray Spectrom. 13 (1984) 91-7.

[17] Bastin G. F., HeIJligers H. J. M. and VAN LOO F. J. J., Scanning 6 (1984) 58-68.

[18] BASTIN G. F. and HEIJLIGERS H. J. M. Scanning 8 (1986) 45-67.

[19] Bastin G. F. and HeiJuigers H. J. M. Quantitative Electron Probe Microanalysis of Boron in Binary Borides. Report of the University of Technology Eindhoven, Laboratory for Physical Chemistry, PO Box 513, NL-5600 MB Eindhoven, ISBN 90-6819-006-7 CIP (1986).

[20] BASTIN G. F. and HeIJligers H. J. M. in: Proc. 1st EMAS Workshop (Antwerpen 1989), (the European Microbeam Analysis Society, Antwerpen, 1989) 81-106.

[21] TIRIRA SAÁ J. H. and RIVEROS J. A., X-ray Spectrom. 16 (1987) 27-32.

[22] Tirira SAÁ J. H. Del Giorgio M. A. and Riveros J. A., X-ray Spectrom. 16 (1987) 243-8.

[23] REHBACH W., Ph D Thesis, RWTH Aachen (1987).

[24] REHBACH W. and KARDUCK P., Microbeam Analysis 1988, Newbury D. E. Ed. (San Francisco Press, San Francisco, 1988) 285-9.

[25] GAUTSCHI W., in: Handbook of Mathematical Functions, Abramowitz M. and Stegun I. A. Eds. (Dover Publications, New York, 1965) 295-330.

[26] HEINRICH K. F. J. Electron Beam X-ray Microanalysis, van Nostrand Reinhold Co., New York (1981).

[27] FINK R. W., Rev. Mod. Phys. 38 (1966) 513-40.

[28] Bethe H. A., Ann. Phys. 5 (1930) 325-400. 